\title{
Comparative Structural Analysis of Oxidized and Reduced Thioredoxin from Drosophila melanogaster
}

\section{Markus C. Wahl ${ }^{1 *}$, Angelika Irmler ${ }^{2}$, Beate Hecker ${ }^{2}$, R. Heiner Schirmer ${ }^{3}$ and Katja Becker ${ }^{2 *}$}

\author{
${ }^{1}$ Max-Planck-Institut für \\ Biophysikalische Chemie \\ Arbeitsgruppe \\ Röntgenkristallographie, Am \\ Faßberg 11, D-37077 Göttingen \\ Germany \\ ${ }^{2}$ Interdisziplinäres \\ Forschungszentrum \\ Justus-Liebig-Universität \\ Giessen, Heinrich-Buff-Ring \\ 26-32, D-35392 Giessen \\ Germany \\ ${ }^{3}$ Biochemie-Zentrum \\ Ruprecht-Karls-Universität \\ Heidelberg, Im Neuenheimer \\ Feld 504, D-69120 Heidelberg \\ Germany
}

\begin{abstract}
Thioredoxins (Trx) participate in essential antioxidant and redox-regulatory processes via a pair of conserved cysteine residues. In dipteran insects like Drosophila and Anopheles, which lack a genuine glutathione reductase (GR), thioredoxins fuel the glutathione system with reducing equivalents. Thus, characterizing Trxs from these organisms contributes to our understanding of redox control in GR-free systems and provides information on novel targets for insect control. Cytosolic Trx of Drosophila melanogaster (DmTrx) is the first thioredoxin that was crystallized for X-ray diffraction analysis in the reduced and in the oxidized form. Comparison of the resulting structures shows rearrangements in the active-site regions. Formation of the $\mathrm{C} 32-\mathrm{C} 35$ disulfide bridge leads to a rotation of the sidechain of C32 away from C35 in the reduced form. This is similar to the situation in human $\operatorname{Tr} x$ and $\operatorname{Tr} x \mathrm{~m}$ from spinach chloroplasts but differs from Escherichia coli Trx, where it is C35 that moves upon change of the redox state. In all four crystal forms that were analysed, DmTrx molecules are engaged in a non-covalent dimer interaction. However, as demonstrated by gel-filtration analyses, DmTrx does not dimerize under quasi in vivo conditions and there is no redox control of a putative monomer/dimer equilibrium. The dimer dissociation constants $K_{\mathrm{d}}$ were found to be $2.2 \mathrm{mM}$ for reduced DmTrx and above $10 \mathrm{mM}$ for oxidized DmTrx as well as for the protein in the presence of reduced glutathione. In human Trx, oxidative dimerization has been demonstrated in vitro. Therefore, this finding may indicate a difference in redox control of GR-free and GR-containing organisms.
\end{abstract}

(C) 2004 Elsevier Ltd. All rights reserved.

Keywords: dimerization; Drosophila melanogaster; redox regulation; thioredoxin; X-ray crystal structure

\section{Introduction}

The cellular defense against reactive oxygen species includes the glutathione (GSH) and the thioredoxin (Trx) redox systems. The abundant tripeptide GSH is present in millimolar concentrations and contributes largely to the intracellular $\mathrm{SH}$ buffer. The ubiquitous $12 \mathrm{kDa}$ protein Trx comprises general antioxidative properties as well as highly specialized functions such as the

Abbreviations used: $\beta$-ME, $\beta$-mercaptoethanol; GR, glutathione reductase; GSH, reduced glutathione; GSSG, oxidized glutathione; IPTG, isopropyl- $\beta$-Dthiogalactopyranoside; TCEP, Tris(2-carboxyethyl)phosphine; Trx, thioredoxin; $\operatorname{Tr} x \mathrm{R}$, thioredoxin reductase.

E-mail addresses of the corresponding authors: mwahl@gwdg.de; becker.katja@gmx.de involvement in deoxyribonucleotide production, redox control of different metabolic pathways, cell proliferation and differentiation, embryogenesis as well as tumor growth and apoptosis. ${ }^{1-4}$ In most organisms, oxidized $\operatorname{Trx}\left(\operatorname{Tr} x-\mathrm{S}_{2}\right)$ and glutathione disulfide (GSSG) are converted to their reduced thiol forms (Trx-SH ${ }_{2}$, GSH) by the NADPH-dependent flavoenzymes thioredoxin reductase (TrxR) and glutathione reductase (GR), respectively., Recently, however, dipteran insects like Drosophila melanogaster and Anopheles gambiae were found to lack a genuine GR. ${ }^{7,8}$ GSSG reduction has been shown to be effected non-enzymatically by Trx- $\mathrm{SH}_{2}$ in these organisms. Although this reaction has been demonstrated for thioredoxins of other organisms like man, the malarial parasite Plasmodium falciparum and Escherichia coli, ${ }^{9}$ it is most likely to be of particular importance in GR-deficient systems. 
Therefore, features distinguishing thioredoxins of dipteran insects and other organisms lacking a GR, like trypanosomes, ${ }^{10}$ from thioredoxins of GRcontaining organisms are of great interest with regard to mechanisms of redox reactions and their control. In A. gambiae, as well as in D. melanogaster, Trx- $S_{2}$ is reduced by the $C$-terminal redox center of a high molecular mass TrxR. As shown for Drosophila, cytosolic and mitochondrial TrxR isoforms are essential for viability and represent splicing variants of one $\operatorname{tr} x r 1$ gene. $^{11}$

Like other organisms, Drosophila possesses several thioredoxins that differ in their intracellular localization and their functions. The ovary-specific DmTrx1, which is also called DHD (deadhead) protein, and the testis-specific TrxT are both localized in the nucleus and are arranged as a gene pair. ${ }^{12}$ The cytosolic DmTrx, also called DmTrx2, represents the metabolically prominent thioredoxin and is the preferred substrate of thioredoxin peroxidase. ${ }^{13}$ Despite the different numbering, the physiological function of $\operatorname{Tr} x 2$ in Drosophila is analogous to that of Trx1 in human. Therefore, DmTrx2 is called DmTrx in the following. Furthermore, a dimeric protein related to disulfide isomerases, named WIND, is located in the endoplasmatic reticulum. ${ }^{14}$ In humans, eight thioredoxins have been described so far, among them the cytosolic Trx1 with the enzymatically inactive truncated form Trx80 and the splicing variant delta3Trx1, the mitochondrial $\operatorname{Tr} x 2$ and three sperm-specific SpTrxs. ${ }^{15-20}$

In spite of the many and diverse functions, the 3D structures of thioredoxins are highly conserved. The Trx fold consists of five central $\beta$-strands surrounded by four $\alpha$-helices. The typical active-site motif C32-G-P-C35 (nomenclature of human Trx1 and DmTrx) resides between the $\beta-2$ strand and the $\alpha-2$ helix. The thiol of C32 has a low $p K_{a}$ value and the thiolate group is the attacking nucleophile in protein disulfide reduction. During catalysis, a mixed disulfide transition state is formed, followed by a reversible and fast thiol-disulfide exchange in a hydrophobic microenvironment. ${ }^{21,22}$ The oxidizing and reducing properties of human Trx1 are directed by several factors. The S-nitrosylation of C69 has been shown to be essential for the redox regulatory and the apoptotic function of $\operatorname{Trx}$ in endothelial cells. ${ }^{23}$ Under oxidative stress, C73 is reversibly glutathionylated, which reduces the enzymatic activity of human Trx. ${ }^{24} \mathrm{~A}$ second disulfide motif, C62-C69 has been identified in human Trx1 that can transiently impair Trx activity during redox signalling or oxidative stress; this allows more time for sensing and transmission of oxidative signals. ${ }^{25}$ Since DmTrx lacks C69 as well as C73 equivalents, these regulatory processes are unlikely to occur in D. melanogaster. Under oxidizing conditions, human Trx forms disulfide-linked homodimers via $\mathrm{C} 73 .^{22,26}$ Under strongly reducing conditions and low $\mathrm{pH}$, non-covalently linked dimers are formed, with particular involvement of the interface residues D60 and W31. ${ }^{27}$ Human Trx dimers are inactive, and dimer formation is therefore discussed as a means of regulating Trx activity.

Here, we present the crystal structures of oxidized and reduced cytosolic Trx from D. melanogaster (DmTrx). The molecular architecture of the protein is similar in both redox states and resembles that of other thioredoxins analyzed previously. In the crystals, DmTrx is present as a dimer. However, as revealed by comparative gelfiltration analyses, a dimerization of DmTrx is most unlikely to be of physiological relevance and, in contrast to human Trx, cannot be regulated by the redox environment. This lack of inactivation by peripheral oxidation and dimerization underlines the importance of the thioredoxin system in organisms lacking a genuine glutathione reductase.

\section{Results and Discussion}

\section{Overall structures}

Recombinant DmTrx could be crystallized readily under various conditions without removal of its $\mathrm{N}$-terminal $\mathrm{H}_{6}$-tag. Employing protein that had been purified under oxidizing conditions, three different crystal forms were identified that yielded diffraction data between $1.8 \AA$ and $2.1 \AA$ resolution (Table 1). All three crystal structures were solved by molecular replacement using the structure coordinates of oxidized human $\operatorname{Tr} x^{22}$ and were refined including data up to $1.9 \AA, 2.2 \AA$ and $2.3 \AA$ resolution, respectively (Table 1 ). Each structure revealed four Trx molecules per asymmetric unit. A fourth crystal form was obtained upon treatment of the protein with the reducing agent Tris(2carboxyethyl)-phosphine (TCEP; $10 \mathrm{mM}$ ) prior to crystallization ( $2.3 \AA$ resolution). This structure was solved also by rotation/translation searches (Table 1) and exhibited a single polypeptide per asymmetric unit. All structures were refined by standard protocols, in which $5 \%$ of the reflections were used to continuously monitor the free $R$-factor. The refined structures maintained excellent geometry and showed only a small number of outliers in Ramachandran plots. All protein molecular models encompassed the entire 106 residues of DmTrx.

Like other thioredoxins, ${ }^{28}$ DmTrx consists of a central five-stranded mixed $\beta$-sheet (strands $\beta 1-\beta 5$ ) capped on each side by two $\alpha$-helices $(\alpha 1 / 3$ and $\alpha 2 / 4$, respectively; Figure $1 \mathrm{~A})$. The redox active cysteine pair (C32 and C35) is situated at the very $\mathrm{N}$ terminus of helix $\alpha 2$. In the absence of a reducing agent, these two cysteine residues are clearly engaged in a disulfide bridge (DmTrx-S ${ }_{2}$; Figure 1B; crystal forms $1-3$ of Table 1 ). Conversely, pretreatment with TCEP led to the crystallization of the reduced form of DmTrx in crystal form 4 (DmTrx-(SH) $)_{2}$; Figure 1B; Table 1).

C35 of the redox active cysteine pair is covered largely by the C-terminal portion of helix $\alpha 2$ (Figure 1A). In addition, C32 and C35 or the 
Table 1. Crystallographic data

\begin{tabular}{|c|c|c|c|c|}
\hline & & Oxidized & & Reduced \\
\hline \multicolumn{5}{|l|}{ A. Crystallization } \\
\hline Crystal form & 1 & 2 & 3 & 4 \\
\hline Crystallization buffer & $\begin{array}{c}\mathrm{pH} 4.6,0.1 \mathrm{M} \mathrm{CdCl}_{2} \\
\text { 30\% PEG400 }\end{array}$ & $\begin{array}{c}\mathrm{pH} \text { 5.2, } 0.1 \mathrm{M} \mathrm{CdCl}_{2} \\
16 \% \text { PEG400 }\end{array}$ & $\begin{array}{c}\mathrm{pH} 6.0,0.2 \mathrm{M} \mathrm{AS},{ }^{\mathrm{a}} 25 \% \\
\text { PEG4000 }\end{array}$ & $\mathrm{pH} 8.5,2.1 \mathrm{M} \mathrm{AS}$ \\
\hline \multicolumn{5}{|l|}{ B. Data collection } \\
\hline Radiation source & Synchrotron & Rotating anode & Rotating anode & Rotating anode \\
\hline Wavelength $(\AA ̊ \cap)$ & 1.05 & 1.5418 & 1.5418 & 1.5418 \\
\hline Space group & $P 4_{1} 2_{1} 2$ & $P 4_{2} 2_{1} 2$ & $P 2_{1}$ & $\mathrm{P6}_{5} 22$ \\
\hline \multicolumn{5}{|l|}{ Unit cell parameters } \\
\hline$a(\AA)$ & 92.5 & 99.6 & 60.7 & 52.9 \\
\hline$b(\AA)$ & 92.5 & 99.6 & 65.5 & 52.9 \\
\hline$c(\AA)$ & 97.6 & 88.0 & 62.1 & 153.0 \\
\hline$\beta$ (deg.) & & & 113.1 & \\
\hline Resolution $(\AA)$ & $20.0-1.8$ & $30.0-2.1$ & $30.0-2.0$ & $30.0-2.3$ \\
\hline \multicolumn{5}{|l|}{ Reflections } \\
\hline Unique & 37,273 & 22,855 & 20,371 & 5951 \\
\hline Redundancy & 2.8 & 5.3 & 3.5 & 3.5 \\
\hline Completeness (\%) & $94.1(88.1)$ & $86.8(91.5)$ & $98.4(95.6)$ & $95.8(95.6)$ \\
\hline$I / \sigma(I)$ & $21.1(1.3)$ & $17.2(2.1)$ & $21.4(3.5)$ & $12.9(1.9)$ \\
\hline$R_{\text {sym }}$ & $0.038(0.641)$ & $0.042(0.561)$ & $0.071(0.323)$ & $0.070(0.590)$ \\
\hline \multicolumn{5}{|l|}{ C. Refinement } \\
\hline Resolution $(\AA)$ & 20.0-1.9 & 20.0-2.2 & 20.0-2.3 & $20.0-2.3$ \\
\hline No. reflections (\%) & $32,287(95.0)$ & $18,759(86.2)$ & $18,184(90.8)$ & $5921(95.9)$ \\
\hline Test set (\%) & 5 & 5 & 5 & 10 \\
\hline$R_{\text {work }}{ }^{c}$ & 0.234 & 0.226 & 0.219 & 0.220 \\
\hline$R_{\text {free }}{ }^{c}$ & 0.269 & 0.288 & 0.261 & 0.259 \\
\hline \multicolumn{5}{|l|}{ Contents of a.u. ${ }^{\mathrm{d}}$} \\
\hline Protein molecules & 4 & 4 & 4 & 1 \\
\hline Protein atoms & 3309 & 3284 & 3284 & 821 \\
\hline Water atoms & 418 & 218 & 271 & 42 \\
\hline Ligands & $3 \mathrm{Cd}^{2+} 1 \mathrm{Cl}^{-}$ & $3 \mathrm{Cd}^{2+}$ & - & - \\
\hline \multicolumn{5}{|l|}{ Mean $B$-factors $\left(\AA^{2}\right)$} \\
\hline Wilson & 35.0 & 34.4 & 37.3 & 47.1 \\
\hline Protein & 43.6 & 23.0 & 32.0 & 52.2 \\
\hline Water & 55.8 & 42.4 & 35.8 & 68.3 \\
\hline Ligands & 36.4 & 52.5 & - & - \\
\hline \multicolumn{5}{|l|}{ Ramachandran plot (\%) } \\
\hline Preferred & 98.2 & 99.0 & 97.4 & 98.9 \\
\hline Additionally allowed & 0.8 & 0.5 & 0.8 & 0.0 \\
\hline Disallowed & 1.0 & 0.5 & 1.8 & 1.0 \\
\hline \multicolumn{5}{|l|}{ rmsd ${ }^{\mathrm{e}}$ geometry } \\
\hline Bond lengths $(\AA)$ & 0.006 & 0.010 & 0.007 & 0.007 \\
\hline Bond angles (deg.) & 1.10 & 1.08 & 1.23 & 1.19 \\
\hline \multicolumn{5}{|l|}{ rmsd $B$-factors $\left(\AA^{2}\right)$} \\
\hline Main-chain bonds & 1.8 & 3.3 & 1.5 & 1.4 \\
\hline Main-chain angles & 2.4 & 4.4 & 2.4 & 2.5 \\
\hline Side-chain bonds & 2.8 & 3.8 & 2.4 & 2.4 \\
\hline Side-chain angles & 3.8 & 5.0 & 3.4 & 3.7 \\
\hline PDB entry & 1XWA & 1XWB & 1XW9 & 1XWC \\
\hline \multicolumn{5}{|c|}{$\begin{array}{l}\text { Data for the last } 0.1 \AA \text { are given in parentheses. } \\
\text { a } A S \text {, ammonium sulphate. } \\
\text { b } R_{\text {sym }}(I)=\sum_{h k l} \sum_{i}\left[\left|I_{i}(h k l)-\langle I(h k l)\rangle\right|\right] / \sum_{h k l} \sum_{i}\left[I_{i}(h k l)\right] ; I_{\mathrm{i}}(h k l) \text { is the intensity of the } i \text { th measurement of } h k l \text {; } \\
\text { value of } h k l \text { for all } i \text { measurements. } \\
\left.\text { c } R_{\text {work }}=\sum_{h k l}\left[|| F_{\text {obs }}|-| k\left|F_{\text {calc }}\right| \mid\right] / \sum_{h k l}|| F_{\text {obs }} \mid\right] ; R_{\text {free }}=\sum_{h k l \subset T}\left[|| F_{\text {obs }}|-k| F_{\text {calc }}||\right] / \sum_{h k l \subset T}\left[\left|F_{\text {obs }}\right|\right] \text {; } h k l \subset T \text {-test set. } \\
\text { d a.u., asymmetric unit. } \\
\text { e rmsd, root-mean-square deviation. }\end{array}$} \\
\hline
\end{tabular}

corresponding disulfide are laterally shielded from the solvent by the loop between $\beta 2$ and $\alpha 2$, by the $N$ terminus of helix $\alpha 2$, and by the C-terminal part of the $\alpha 3-\beta 4$ loop. This arrangement leaves only one side of the C32 sulfhydryl group accessible to the solvent (at the top in Figure 1A) and the transfer of reducing equivalents. This notion is supported by the structure of the E. coli TrxR-Trx complex (contact zone labelled $\alpha 3$ region in Figure 2). ${ }^{29}$ Similarly, a molecular model predicts that mammalian Trxs approach their reductases via the same region. ${ }^{30}$

\section{Differences in the structures of oxidized and reduced DmTrx}

The global fold of oxidized DmTrx is similar across all three crystal forms and all 12 crystallographically independent molecules, the pair-wise 
A
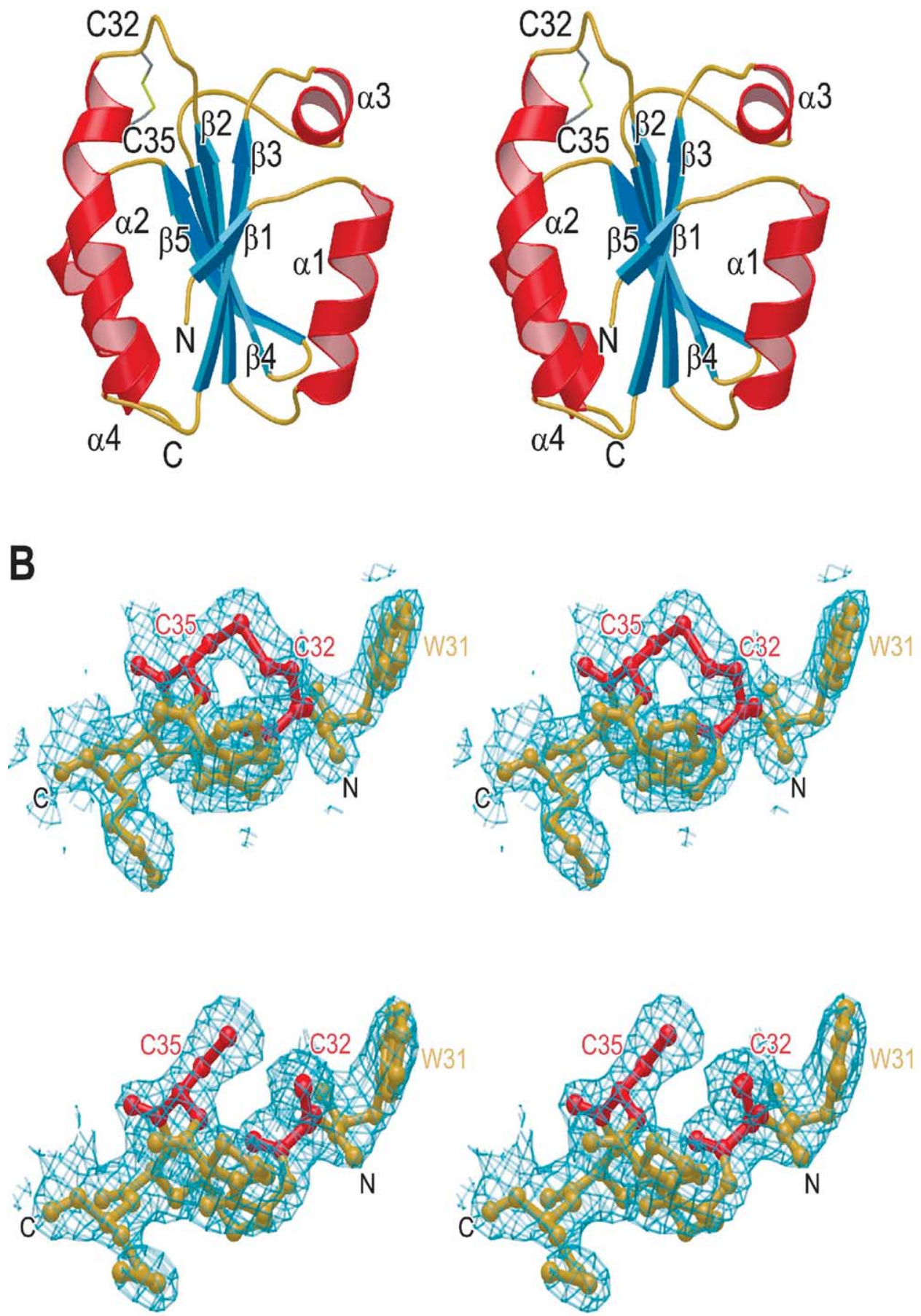

C

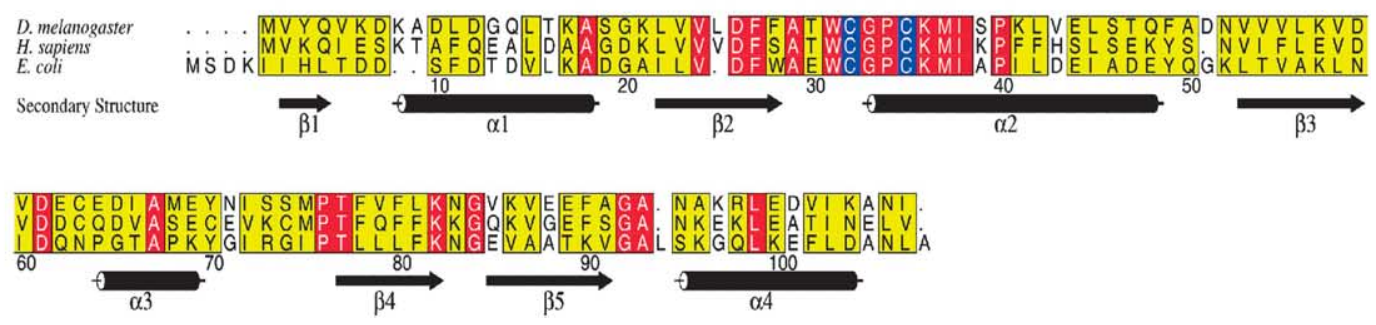

Figure $1 \mathrm{~A}-\mathrm{C}$ (legend opposite) 
D

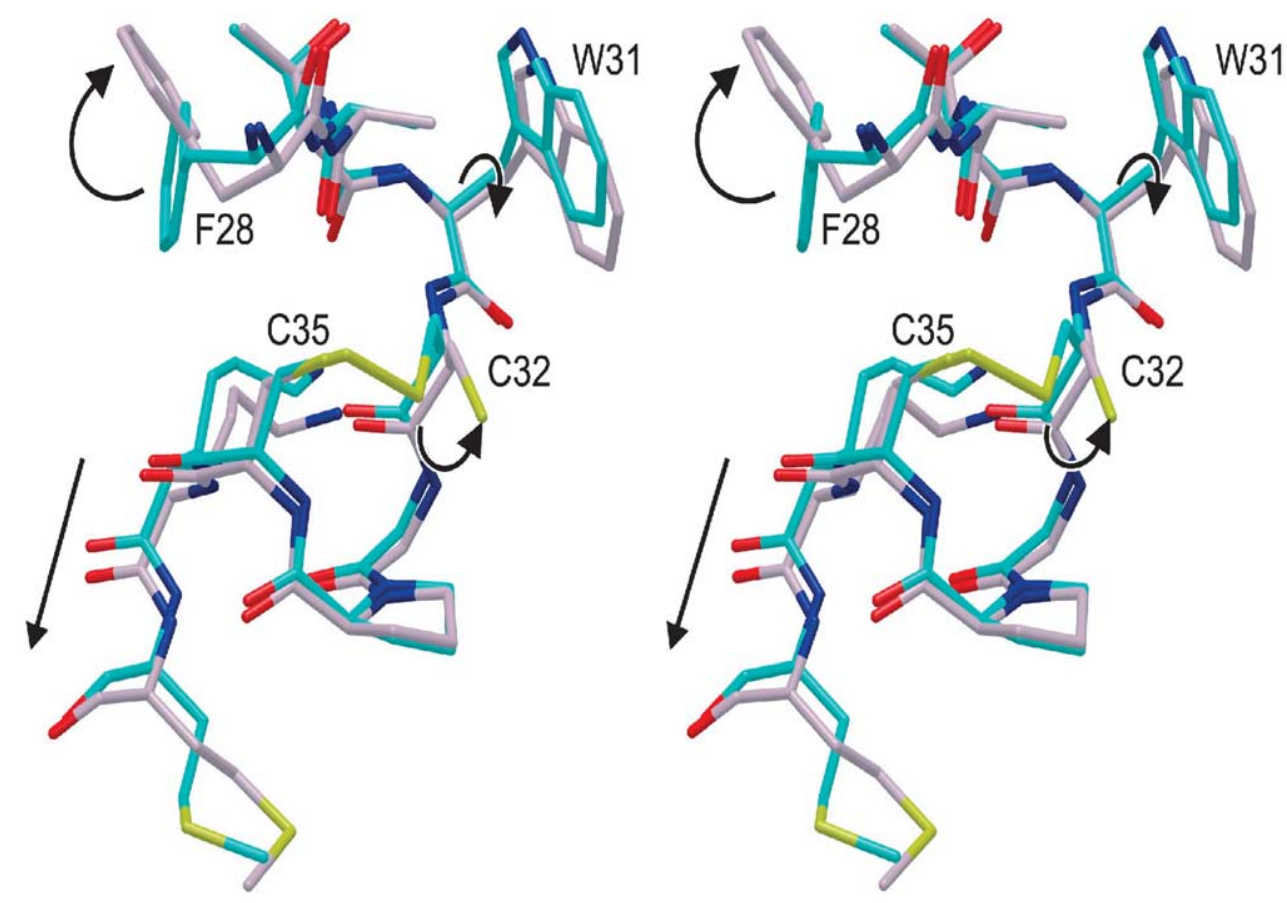

Figure 1. Structures of DmTrx. A, Stereo ribbon plot of oxidized DmTrx. $\alpha$ Helices are in red, $\beta$ strands are in blue and coiled regions are in gold. Helices and strands are numbered from the $\mathrm{N}$ to the $\mathrm{C}$ terminus. The redox-active disulfide, formed by residues C32 and C35, is displayed with sticks (carbon, gray; sulfur, yellow). B, Stereo images of the final $2 F_{\mathrm{o}}-F_{\mathrm{c}}$ electron densities ( $\sigma \sigma$ level) covering the redox-active region (ball-and-stick; redox-active cysteine residues are in red). Relevant residues are labeled. Top panel: oxidized form; bottom panel, reduced form. C, Sequence alignment of DmTrx, human thioredoxin and E.coli thioredoxin. Identical residues are shown on a red background, residues conserved in at least two of the molecules are coded yellow. The redox-active cysteine residues (C32 and C35) are shown in blue. The numbering is according to the DmTrx sequence. The secondary structure elements as observed in the present structures are indicated below the alignment. D, Stereo stick figures of the superimposed active-site regions of oxidized and reduced DmTrx. The molecules are color-coded by atom type. Carbon atoms of the reduced form are in a pinkish-gray, those of the oxidized form are in cyan. Arrows indicate possible side-chain and backbone rearrangements, which are observed upon transition from the oxidized to the reduced state.

root-mean-square deviation (rmsd) for all $106 \mathrm{C}^{\alpha}$ atoms being $\sim 0.25 \AA$. Local conformational differences are largely limited to the side-chains of surface-exposed residues. While reduced DmTrx maintains the same overall fold (rmsd to oxidized forms for all $106 \mathrm{C}^{\alpha}$ atoms $\sim 0.55 \AA$ ), some local rearrangements in the active-site region are discernible when compared to the oxidized form. Significantly, in the present studies DmTrx was reduced prior to crystallization, giving the protein the conformational freedom to pack in a crystal form different from those observed for the oxidized state. Thus, a priori we eliminated the possibility that conformational differences between the redox states were dampened or biased by the same crystal packing contacts.

In the region of the redox active center we observe different side-chain conformations for a number of residues (Figure 1D). In most structures, the aromatic ring of F28 is oriented away from the cysteine pair but in two oxidized molecules it is seen to swing over C35. While there is no strict bimodal distribution of the F28 conformers, which correlates with the redox state of DmTrx, only molecules from the oxidized ensemble show F28 covering C35. This residue may thus serve to block movement of C35 upon approach of the C32 sidechain during oxidation. The indole ring of W31 moves closer to $\mathrm{C} 32$ in the reduced form, thus engaging in van der Waals contacts to C32 (Figure 1D). A similar situation was seen with human Trx. ${ }^{22}$ While C35 is oriented similarly in the oxidized and reduced structures, a rotation around the $C^{\alpha}-C^{\beta}$ bond is observed in $C 32$, which in the reduced protein turns the C32 sulfhydryl group away from the side-chain of C35 and closer to the side-chain of W31 (Figure 1D). The distance between the two sulfur atoms is thus increased from $2.07 \AA$ to $3.85 \AA$ upon reduction. One can therefore envisage a concerted motion of the C32 sulfhydryl group and the W31 indole ring which, by attractive interactions, supports withdrawal of C32 from C35 upon reduction. Finally, P34 shows various ring puckers in the crystallographically independent molecules, which are, however, not correlated with the redox state (not shown).

In addition, slight rearrangements in the backbone region around the active center are observed 
A
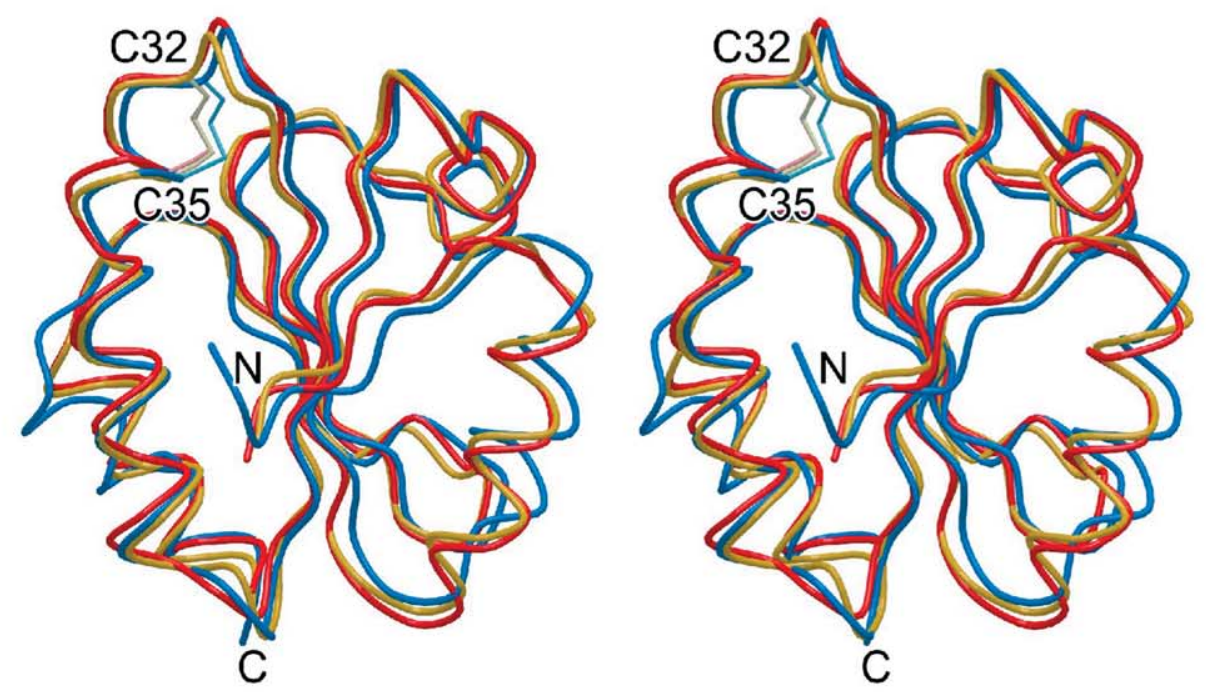

B
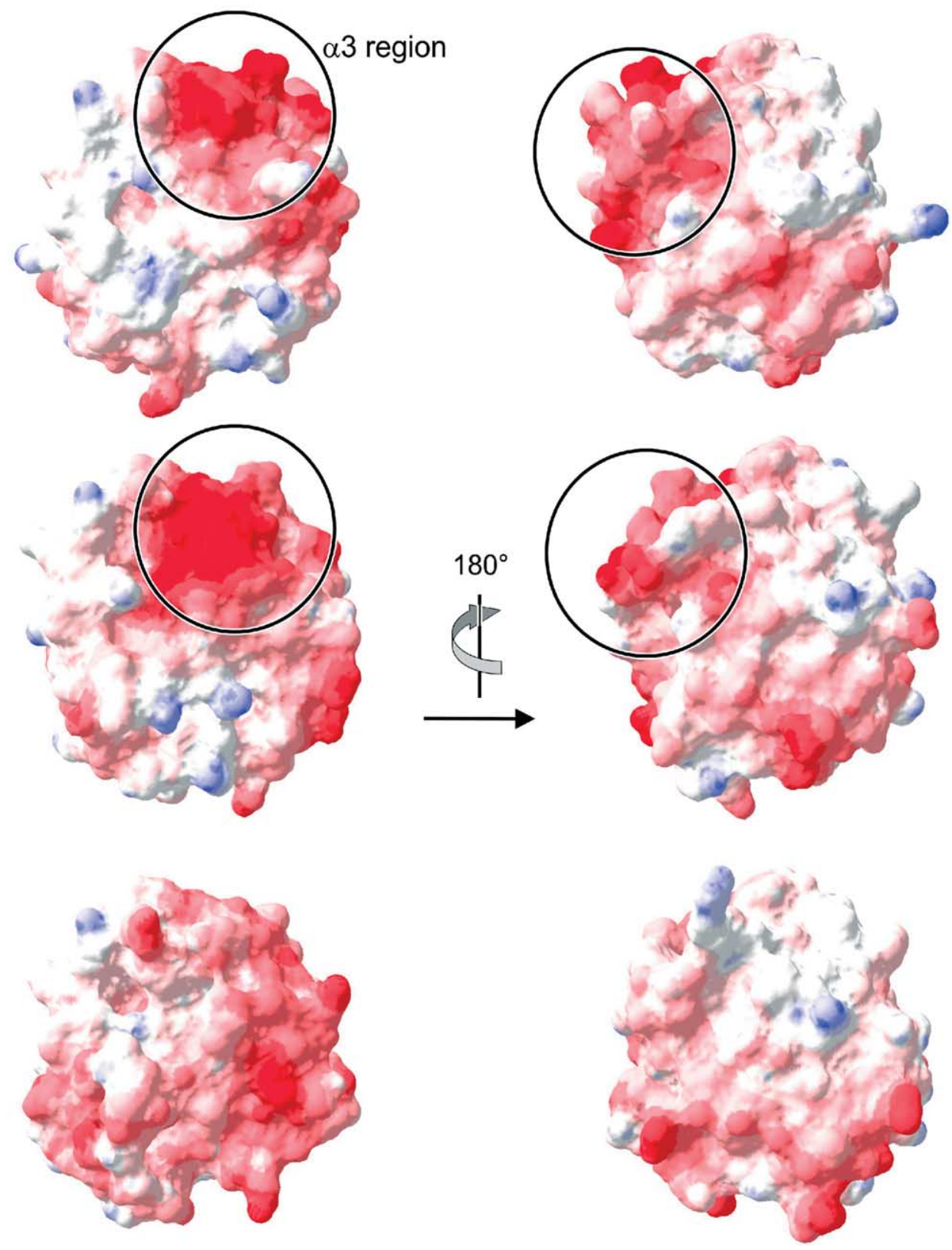

Figure 2 (legend opposite) 
upon change of the redox state. In DmTrx- $S_{2}$, the $C^{\alpha}$ atoms of C32 and C35 approach each other by $\sim 0.7 \AA$ thus tightening the redox active loop (C32G33-P34-C35; Figure 1D). Furthermore, closure of the disulfide bridge "pulls" the backbone of residues C-terminal to C35 (K36-I38) closer to the redox center each by about $\sim 0.5 \AA$. The residues $\mathrm{N}$-terminal of C32 are less affected. Thus, oxidation is accompanied by a general tightening of the redox loop and of the region C-terminal to the active center.

From the above observations, a possible scenario for the reduction of DmTrx includes a movement of the C32 side-chain by a simple rotation about the $C^{\alpha}-C^{\beta}$ bond towards the protein surface. This movement may be supported by a concomitant engagement of its $C^{\beta}$ and $S^{\gamma}$ atoms in van der Waals contacts with the indole ring of W31. Upon oxidation, attack of C35 by the C32 thiolate group may be supported by fixation of the C 35 orientation with the side-chain of F28. In the reduced form, the $\mathrm{p} K_{\mathrm{a}}$ of C32 is most likely decreased by the sulfhydryl group of C35, which is within hydrogen bonding distance from the $S^{\gamma}$ of $C 32$, similar to the situation found in human Trx. ${ }^{22}$ No other ionizable group or helix pole is discernible in the vicinity of C32, which could influence its $\mathrm{p} K_{\mathrm{a}}$ and thus favor the thiolate form relative to the thiol form.

\section{Comparison to thioredoxins from other species}

The overall fold of DmTrx resembles closely those observed for other thioredoxins (Figure 2A; rmsd DmTrx versus human Trx $\sim 0.70 \AA$; rmsd DmTrx versus E. coli $\operatorname{Trx} \sim 1.2 \AA$ ). This is consistent with high levels of sequence identity, in particular around the active site (Figure 1C). Like other eukaryotic thioredoxins, DmTrx exhibits a negatively charged surface patch around helix $\alpha 3$ ( $\alpha 3$ region in Figure 2B), which is employed in making contact with thioredoxin reductase and with the target molecules, upon which reduced Trx acts. ${ }^{29-32}$ E. coli Trx has a different surface charge pattern, reflecting the fact that bacterial TrxR differs fundamentally from insect TrxR.

Similar to our results, reduced and oxidized structures of bacterial, ${ }^{31,33}$ spinach chloroplast $^{32}$ and human ${ }^{22}$ thioredoxins were found to lack global conformational differences. As demonstrated previously by NMR, the conformational change between oxidized and reduced human thioredoxin mutants (C62A, C69A, C73A) is very subtle and restricted to areas in spatial proximity to the redox active cysteine residues. ${ }^{34}$ However, the local changes encompassing the active-site loop observed when comparing the crystal structure of oxidized E. coli $\operatorname{Trx}^{31}$ and the NMR structure of reduced E. coli $\operatorname{Tr}^{33}$ appear to be more pronounced and of a different nature than the rearrangements seen in DmTrx. In particular, the movements of residues G33 and P34 were found rather small in the present work compared to the E. coli structures. The movement of the F28 side-chain, observed in DmTrx in a subset of the oxidized molecules, is not observed in the bacterial Trx. Both the backbone and the side-chain of C35 of E. coli Trx seem to undergo a considerable relocation upon change of the redox state, while C32 remains largely fixed. The opposite is seen in DmTrx. Part of these differences between the bacterial and the insect systems may be explained by the structure determination strategies employed for E. coli Trx. However, they may also be a signature of the different TrxRs. ${ }^{6}$ DmTrxR belongs to the high molecular mass TrxRs containing an additional C-terminal redox center located on a flexible loop. In contrast, the E. coli protein is a low molecular mass TrxR with $35 \mathrm{kDa}$ per subunit; a domain rotation is required in this protein to allow electron transport from NADPH to FAD, and from FAD to the active-site disulfide, which then reduces the substrate thioredoxin. ${ }^{6}$

In line with the present results on DmTrx, a rotational movement of the side-chain of the first active-site loop cysteine residue constitutes the main difference between oxidized and reduced Trx from man and spinach chloroplasts. ${ }^{2,32}$ It should be noted that reduced human Trx was crystallized and then allowed to oxidize over a period of several months; ${ }^{22}$ on the other hand, the structure of reduced $\operatorname{Trx} \mathrm{m}$ was obtained by reduction of oxidized crystals. ${ }^{32}$ Thus, structural changes in the redox states of both proteins may have been dampened by crystal forces.

\section{Dimer formation}

Interestingly, all 13 crystallographically independent DmTrx molecules are engaged in a conserved dimer interaction with neighboring molecules (Figure 3), either within the asymmetric units (oxidized forms) or with symmetry-related neighbors (reduced form). The two molecules in the dimers are related by a local or a crystallographic 2-fold symmetry axis, which leads to the orientation of the two $\alpha 3-\beta 4$ loops in an antiparallel $\beta$-arrangement, a contact between the two $\beta 3-\alpha 3$

Figure 2. Phylogenetic comparisons. A, Stereo plot of the superimposed structures of oxidized DmTrx (yellow), human thioredoxin (red) and E. coli thioredoxin (blue). The molecules are oriented as in Figure $1 \mathrm{~A}$. The $\mathrm{N}$ and $\mathrm{C}$ termini and the active-site cysteine residues (sticks in lighter colors) are indicated. B, Electrostatic potential of DmTrx (top), human thioredoxin (center) and E. coli thioredoxin (bottom) mapped onto the protein surface. Red indicates negatively charged regions, blue denotes positively charged areas. The view in the left panels is the same as in A and in Figure 1A. The molecules in the right-hand panels are rotated by $180^{\circ}$ as indicated. A region of strong negative potential surrounding helix $\alpha 3$ is highlighted. This negative region is present in the eukaryotic thioredoxins but not in the bacterial protein. 
A
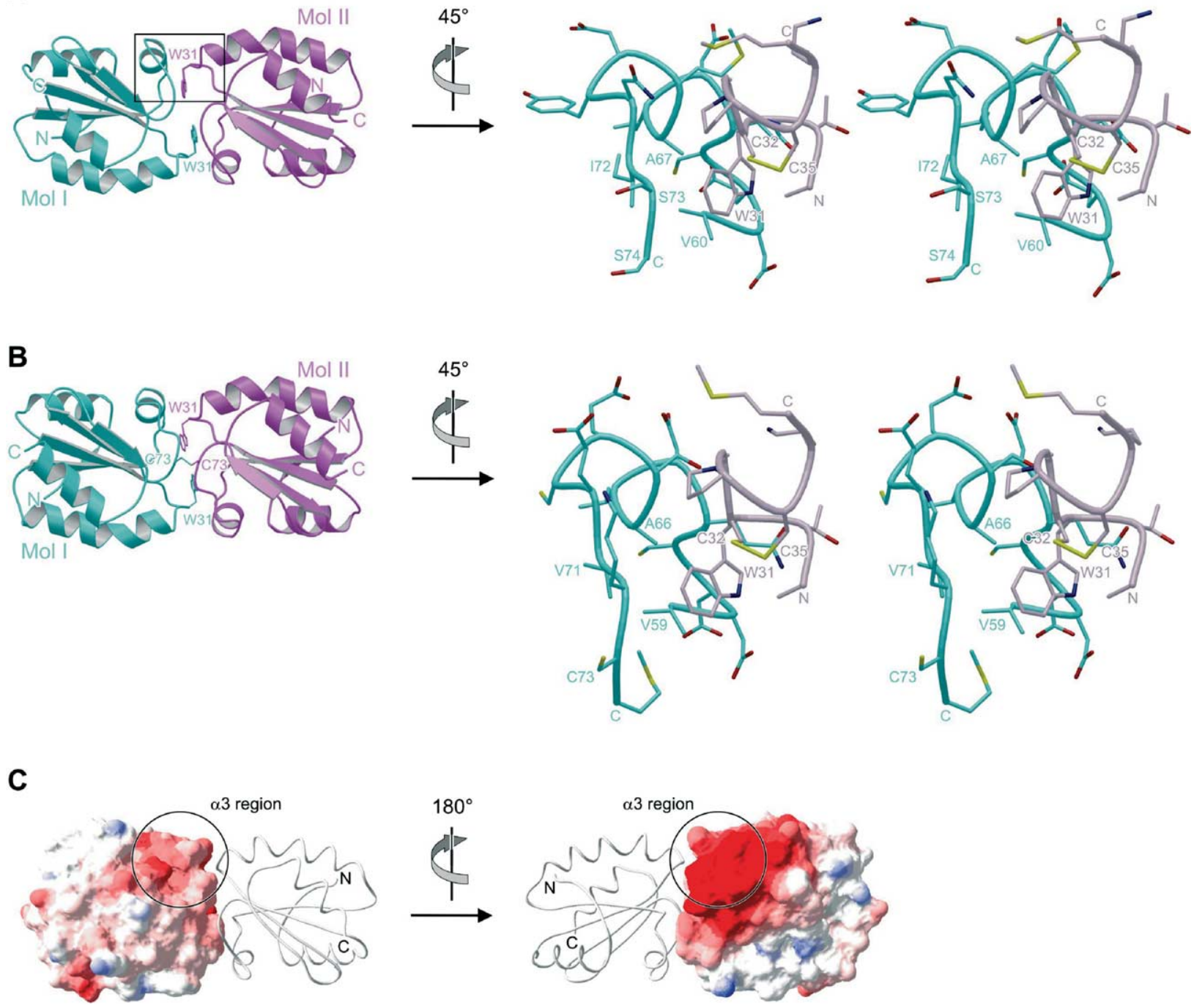

Figure 3. DmTrx dimers. A, Dimers seen in the DmTrx structures. B, Dimers seen in the structures of human Trx. ${ }^{22}$ In both $\mathrm{A}$ and $\mathrm{B}$, one monomer is shown in cyan, the other in magenta. Left-hand panels: overviews of the dimer arrangements. Right-hand panels: details of the interactions. The views in the right-hand panels correspond to $45^{\circ}$ clockwise rotations around the vertical axis compared to the orientations in the left-hand panels (indicated). Critical residues and the protein termini are labeled. The side-chains of important residues are displayed as sticks. Residues in the right-hand panels are color-coded by atom type, with carbon atoms maintaining the colors of the respective protomers (left-hand panels). The backbone traces of the right-hand panels are depicted by smooth tubes, which are colored according to the structures in the left-hand panels. C, Diametric views on a DmTrx dimer with one protomer in an electrostatic surface representation and the other shown as a smooth tube (gray). The orientation in the left-hand panel corresponds to the orientation of the left-hand panel of A. The protein termini and the $\alpha 3$ region are indicated.

loops and the mutual binding of the $\beta 2-\alpha 2$ loop of one molecule to helix $\alpha 3$ and the neighboring loops of the other. As a result of these approaches, the side-chain of W31 of one molecule is nestled in a hydrophobic cavity formed by residues V60, A67, M68, I72 and M75 of the other subunit (Figure 3, right-hand panels) and vice versa. The C-terminal portion of helix $\alpha 3$ and the following $\alpha 3-\beta 4$ loop, in particular the backbone regions of S73 and S74, thereby effectively bury the side of C32 of the other monomer, which is accessible to the solvent in the monomeric form (see above).

The solution structure solved by NMR spectroscopy of human Trx complexed with its target peptides of NF kappa B and Ref-1 revealed that the binding area is formed by the active-site residues as well as by helices $\alpha 2, \alpha 3$ and $\alpha 4$, strands $\beta 3$ and $\beta 4$ and helix $\alpha 3$, strands $\beta 3$ and $\beta 5$, and the loop between strands $\beta 3$ and $\beta 4$, respectively. Therefore, a region similar to the dimerization area is in contact with the target peptides. ${ }^{35,36}$ Dimerisation would thus impede the substrate binding.

Human Trx can form disulfide-linked dimers through its non-conserved surface-exposed C73 residue, irrespective of the state of the redox-active cysteine pair C32-C $35 .{ }^{22,27}$ Significantly, although C73 is replaced by a serine residue (S74) in the Drosophila protein and the interactions are thus 
non-covalent, the DmTrx dimers are virtually identical with the human Trx dimers (Figure 3). Very similar non-covalent dimers have been described for Chlamydomonas reinhardtii $\operatorname{Trx} \mathrm{h}$ in the oxidized form (not shown). ${ }^{37}$

In contrast to the hTrx dimers found in crystals and in the oxidized protein, no intermolecular association was found in the hTrx variants (C62A, C69A, C73A, M74T and C62A, C69A, C73A) using NMR spectroscopy and analytical ultracentrifugation. ${ }^{38}$ These findings are likely to be due to the different analytical methods and the inserted mutations preventing dimerization.

\section{Dimerization in solution; comparisons of human Trx with DmTrx}

In order to study the oligomerization potential of DmTrx in solution, we conducted analytical gelfiltration studies under different conditions (Table 2). All analyses were carried out in direct comparison with human $\operatorname{Tr} x$ and allowed the assessment of the monomer/dimer ratio. The dilution factor for the monomeric thioredoxins was $33 \pm 3$ at $\mathrm{pH} 7.5$ as judged from the width at half-height of the peak divided by the sample load volume. When a $1.5 \mathrm{mM}$ sample had been applied, the resulting final concentration was $45 \mu \mathrm{M}$, which is close to the estimated cytosolic concentration of $50 \mu \mathrm{M}$ DmTrx. ${ }^{13}$

Under standard conditions (50 mM Tris ( $\mathrm{pH} 7.5)$, $200 \mathrm{mM} \mathrm{NaCl}, 1.5 \mathrm{mM}$ protein) oxidized DmTrx eluted in a sharp monomeric peak at $V_{\mathrm{e}}=$

Table 2. Comparative analysis of human Trx and DmTrx dimerization by gel-filtration

\begin{tabular}{|c|c|c|}
\hline \multirow[b]{2}{*}{ Conditions $^{\mathrm{a}}$} & \multicolumn{2}{|c|}{$\%$ Dimer } \\
\hline & Human Trx & DmTrx \\
\hline $\mathrm{Ox}$ & 43.5 & 0.6 \\
\hline $\mathrm{Ox}+2 \mathrm{mM}$ GSSG & 53.5 & 0.7 \\
\hline $\mathrm{Ox}^{\mathrm{b}}+0.2 \mathrm{mM} \mathrm{H}_{2} \mathrm{O}_{2}$ & $-/$ & 0.12 \\
\hline $\mathrm{Ox}+10 \mathrm{mM}$ GSH & 7.3 & 0.3 \\
\hline $\mathrm{Ox}+0.5 \mathrm{mM}$ DTT & 12.3 & 0.3 \\
\hline $\mathrm{Ox}+5 \mathrm{mM}$ DTT & 7.8 & $0.2^{\mathrm{c}}$ \\
\hline $\mathrm{Ox}+10 \mathrm{mM} \beta-\mathrm{ME}$ & 12.2 & 3.7 \\
\hline $\mathrm{Ox}+50 \mathrm{mM} \beta-\mathrm{ME}$ & 5.9 & 7.3 \\
\hline $\mathrm{Ox}, 50 \mathrm{mM} \mathrm{NaCl},+50 \mathrm{mM} \beta-\mathrm{ME}$ & 9.3 & 0.5 \\
\hline Red $^{\mathrm{d}}$ & 1.1 & 6.4 \\
\hline Red, $50 \mathrm{mM} \mathrm{NaCl}$ & 2.4 & 0.5 \\
\hline $\mathrm{Ox}, 1 \mathrm{M} \mathrm{NaCl}$ & 28.6 & 1.7 \\
\hline Red, $1 \mathrm{M} \mathrm{NaCl}$ & 2.6 & 0.7 \\
\hline \multicolumn{3}{|c|}{$\begin{array}{l}\text { a The conditions were as follows: } 50 \mathrm{mM} \text { Tris- } \mathrm{HCl}(\mathrm{pH} 7.5 \text { at } \\
\left.25^{\circ} \mathrm{C}\right), 200 \mathrm{mM} \mathrm{NaCl} \text { (unless otherwise stated); } 1.5 \mathrm{mM} \text { (calcu- } \\
\text { lated as monomer) protein was loaded onto a Superdex } 75 \\
\text { column; Ox, protein purified under oxidizing conditions; Red, } \\
\text { protein purified under reducing conditions (see Materials and } \\
\text { Methods). } \\
\text { b The protein solution was applied at a concentration of } \\
0.4 \mathrm{mM} \text {. } \\
\text { c In this experiment the peak representing the monomer } \\
\text { reproducibly exhibited a shoulder containing } 7 \% \text { of the total } \\
\text { protein. } \\
\text { d The protein solution was applied at a concentration of } \\
2.7 \mathrm{mM} \text {. }\end{array}$} \\
\hline
\end{tabular}

$81.8( \pm 1) \mathrm{ml}$ with an apparent molecular mass of $10.3 \mathrm{kDa}$ and up to $0.6 \%$ dimers $\left(V_{\mathrm{e}}=68.6( \pm 1) \mathrm{ml}\right.$, apparent molecular mass of $22.6 \mathrm{kDa})$. Under the same conditions, human Trx showed up to $43.5 \%$ dimers $\left(V_{\mathrm{e}}=69.0( \pm 1) \mathrm{ml}\right.$, and an apparent molecular mass of $22.1 \mathrm{kDa}$ ). Treatment with $2 \mathrm{mM}$ GSSG or $0.2 \mathrm{mM} \mathrm{H} \mathrm{H}_{2} \mathrm{O}_{2}$ did not increase the formation of DmTrx dimers significantly $(0.7 \%$ and $0.12 \%$, respectively). Human Trx dimer formation, however, increased up to $53.5 \%$ in the presence of $2 \mathrm{mM}$ GSSG. The dissociation constant $K_{\mathrm{d}}$ for the dimers of DmTrx was calculated to be $>10 \mathrm{mM}$ even in the presence of oxidants.

Reduction of oxidized protein with $10 \mathrm{mM} \mathrm{GSH}$ for 45 minutes decreased dimers of human $\operatorname{Trx}$ to $7.3 \%$ with DmTrx dimers remaining low at $0.3 \%$. The $K_{\mathrm{d}}$ values under these quasi-physiological reducing conditions were $1.05 \mathrm{mM}$ for human Trx and $29.8 \mathrm{mM}$ for DmTrx. Partial reduction with $0.5 \mathrm{mM}$ DTT showed a decrease of human Trx dimers to $12.3 \%$ and no change in DmTrx dimers $(0.3 \%)$, as expected. Incubation with $5 \mathrm{mM}$ DTT for 45 minutes, however, led to $7.8 \%$ human $\operatorname{Tr} x$ dimers, and $7 \%$ of a DmTrx protein species, that appeared as a flat shoulder of the monomeric peak. This species was more abundant when the protein was purified under reducing conditions. Addition of $10 \mathrm{mM}$ and $50 \mathrm{mM}$ mercaptoethanol ( $\beta$-ME) to the oxidized proteins gave rise to $3.7 \%$ and $7.3 \%$ dimers, respectively. However, this dimerization of reduced DmTrx could be abolished almost completely by lowering the salt concentration from $200 \mathrm{mM} \mathrm{NaCl}$ to $50 \mathrm{mM} \mathrm{NaCl}$ or by raising it to $1 \mathrm{M}$ $\mathrm{NaCl}$. Thus, a low percentage $(<7.3 \%)$ of DmTrx dimers was present only at high concentrations of reducing chemicals (DTT or $\beta$-ME) and at $200 \mathrm{mM}$ $\mathrm{NaCl}$. In the presence of the physiological reductant GSH, dimer formation was not observed.

The dimerization of human Trx in the physiologic range of $\mathrm{pH}$ values is based mainly on the disulfide formation between $\mathrm{C} 73$ and $C 73^{\prime}$. In DmTrx, this residue is absent; thus, non-covalent binding forces would have to effect an oligomerization. As shown by Andersen et al. for reduced human Trx, hydrogen bonds between D60 of two monomers are favoured by acidic $\mathrm{pH}$ and high concentrations of protein. $^{27}$ Under our experimental conditions, oxidized DmTrx was, in contrast to human Trx, almost completely monomeric. Quasi-physiological reduction and different concentrations of salt did not change this. Only high concentrations of artificial reductants induced the discrete formation of dimers. Taken together, these data indicate that DmTrx is most unlikely to form dimers in vivo.

\section{Conclusions}

In contrast to previous investigations of thioredoxins, the present DmTrx was crystallized separately in the oxidized and reduced forms. This allowed study of the structural changes independently from the constraints of a constant crystal 
lattice. The structures demonstrate that oxidation is accompanied by a slight tightening of the redoxactive loop and of the backbone region C-terminal of C35. Movements of the F28 and W31 side-chains support the adoption of the respective oxidation states. The $S^{\gamma}(\mathrm{C} 32)-S^{\gamma}(\mathrm{C} 35)$ distance in the different redox states is governed by a movement of the C32 side-chain, while the conformation of C35 remains largely fixed. Both oxidized and reduced DmTrx form non-covalent dimers in the crystals, which resemble the disulfide-bridged dimers known from the human orthologue.

Human Trx forms dimers in the presence of GSSG and monomers under reducing conditions. This suggests that a regulation of the oligomerization state, and therefore the activity of human Trx, by the intracellular redox milieu is possible. A high GSH/GSSG ratio may activate the human thioredoxin system, whereas an increase in GSSG, indicating oxidative stress, shuts down the Trx/TrxR system, thus saving reducing equivalents for the glutathione system. In the case of DmTrx, the presence of GSH and GSSG did not induce dimer formation. This is shown clearly by the high dimer $K_{\mathrm{d}}$ values of $29.8 \mathrm{mM}$ and $12.8 \mathrm{mM}$, respectively. Notably, the dimer $K_{\mathrm{d}}$ value decreased to $2.2 \mathrm{mM}$ when purifying the protein in the presence of DTT. Although the experimental approaches in their detail can only be compared indirectly, the $K_{\mathrm{d}}$ values for human Trx reported by Anderson et al. $(166 \mu \mathrm{M} \text { at } \mathrm{pH} 8.0 \text { and } 6.1 \mu \mathrm{M} \text { at } \mathrm{pH} 3.8)^{27}$ support the conclusion that dimerization is much more favored in the human protein. In contrast, our data strongly indicate that DmTrx does not dimerize under in vivo conditions, and that its physiologic activity is most unlikely to be regulated via subunit association and dissociation.

The lack of an environment-dependent, regulative monomer-dimer equilibrium in DmTrx presumably represents a consequence of the absence of GR in insects like D. melanogaster and A. gambiae. Down-regulating thioredoxin activity under increasing concentrations of GSSG would be deleterious to a system that reduces its glutathione via thioredoxin. From the perspective of evolution, it is likely that the ancestor of human Trx and DmTrx had the potential of forming homodimers, a property shared by most thioredoxins and Trx-like domains. ${ }^{39}$ In the lineage leading to human Trx, dimerization has been elaborated, becoming one of the mechanisms controlling the activity of the protein.

In dipteran insects, where glutathione reductase is absent, the thioredoxin system has become the major system buffering the thiol-based reducing milieu of the cytosol. For a thiol/disulfide redox buffer that is turned over rapidly and often functions under oxidative stress it is advantageous to be based on a stable, simple monomeric molecule. This would explain why a thioredoxin with an increased dissociation constant has been selected in the evolution of dipteran insects. Under crystallization conditions with protein concentrations in the millimolar range, there is a major proportion of Trx dimers. Accordingly, the crystal structures of DmTrx may represent the vestiges of the ancestral dimer.

\section{Materials and Methods}

\section{Cloning, expression and purification of DmTrx}

$\mathrm{N}$-terminally $\mathrm{His}_{6}$-tagged DmTrx was overexpressed in E. coli as described ${ }^{13}$ and purified over an Ni-NTA (nickel-nitriloacetic acid) column (Qiagen) with a yield of $100 \mathrm{mg}$ of protein per $1 \mathrm{l}$ of culture. Protein purification under reducing conditions was carried out in the presence of 0.2-2 mM dithiothreitol (DTT). Eluted protein fractions were brought to $5 \mathrm{mM}$ DTT immediately. Pure fractions of DmTrx were concentrated to $3-4.5 \mathrm{mM}$, dialysed and used immediately or stored at $-20^{\circ} \mathrm{C}$. Oxidized protein was stored in $25 \mathrm{mM}$ Tris $-\mathrm{HCl}$ ( $\mathrm{pH} 7.5$ ), $100 \mathrm{mM} \mathrm{NaCl}$, reduced protein in $10 \mathrm{mM}$ Tris- $\mathrm{HCl}(\mathrm{pH}$ 7.5), $10 \mathrm{mM}$ DTT. For comparative analyses, wild-type human Trx1 was produced analogously. ${ }^{40}$ The determination of the protein concentration was based on an $\varepsilon_{280 \mathrm{~nm}}$ of $8.61 \mathrm{mM}^{-1} \mathrm{~cm}^{-1}$ for DmTrx-S $\mathrm{S}_{2}$ and of $8.25 \mathrm{mM}^{-1} \mathrm{~cm}^{-1}$ for DmTrx-(SH) $)_{2}$.

\section{Crystallization of DmTrx}

Crystallization of DmTrx was based on homemade and commercial incomplete factorial screens. The experiments were conducted in sitting-drop format at various temperatures. DmTrx-S $S_{2}$ yielded crystals of various morphology under a number of polyethylene glycol (PEG) and ammonium sulfate conditions. The crystals were optimised subsequently by systematically varying the $\mathrm{pH}$ against the concentration of precipitant (Table 1). Crystals of DmTrx-(SH $)_{2}$ were generated using the same methods but including $10 \mathrm{mM}$ TCEP in the crystallizing samples.

\section{Data collection, structure solution and refinement}

Crystals of oxidized DmTrx (forms 1-3; Table 1) were transferred into reservoir solutions containing $\sim 30 \%$ $(\mathrm{v} / \mathrm{v})$ glycerol and shock-frozen in liquid nitrogen. During data collection, they were maintained in a stream of liquid nitrogen (Oxford Cryosystems). Crystals of reduced DmTrx were mounted in a special glass capillary with TCEP-containing mother liquor on one side and were measured at room temperature. Diffraction data were collected on a Bruker-Nonius FR591 rotating anode ( $\mathrm{Cu} \mathrm{K \alpha}$ X-radiation at $50 \mathrm{kV}$ and $100 \mathrm{~mA}$ ), equipped with an MAR345 imaging plate detector (MarResearch), and at beamline BW6 of the Deutsches Elektronensynchrotron, Hamburg. Data were processed with the HKL-package. ${ }^{41}$

One structure of oxidized DmTrx (space group $P 4_{2} 2_{1} 2$ ) was solved by rotation/translation searches with MOLREP $^{42}$ using the structure coordinates of oxidized human Trx1 (PDB ID code 1ERU; 22). Through this strategy, we were able to position four molecules in the asymmetric unit, which showed excellent packing interactions. The molecular models were refined by standard procedures in $\mathrm{CNS}^{43}$ and REFMAC5 ${ }^{42}$ with intermittent manual model building in MAIN $\dagger$ (Table 1). One DmTrx model subsequently served to solve all other crystal

$\dagger$ http://www-bmb.ijs.si/doc/index.html 
structures of the oxidized and reduced protein by similar strategies. Initially, the structures of the crystal forms harbouring more than one monomer per asymmetric unit were restrained by the observed non-crystallographic symmetry, these restraints were released in later rounds of refinement and model building.

\section{Analyses in solution}

DmTrx and human Trx dimerization in solution was investigated by analytical gel-filtration chromatography employing an ÄKTA FPLC system (AmershamPharmacia) and a Superdex-75 HiLoad 16/60 size exclusion column (120 $\mathrm{ml}$ bed volume). Bovine serum albumin $(67 \mathrm{kDa})$, ovalbumin $(43 \mathrm{kDa})$, chymotrypsinogen A ( $25 \mathrm{kDa})$, and RNAse A $(13.7 \mathrm{kDa})$ were taken as molecular mass standards. Blue dextran $(\sim 2000 \mathrm{kDa})$ and water indicated the exclusion volumes of the columns $\left(V_{0}\right)$ and the total volume of the liquid phase $\left(V_{\mathrm{t}}\right)$, respectively. The apparent molecular mass of the thioredoxins $(M)$ was evaluated by plotting $\log (M)$ versus $K_{\mathrm{av}}\left(K_{\mathrm{av}}=\left(V_{\mathrm{e}}-V_{0}\right) /\left(V_{\mathrm{t}}-V_{0}\right)\right.$, where $V_{\mathrm{e}}$ is the observed elution volume; $V_{0}$ is the elution volume of a completely excluded species and $V_{\mathrm{t}}$ is the total volume of the liquid phase. The respective protein concentration was established by diluting a sample from the protein stock in the buffer of choice (see Table 2). The samples were incubated for ten minutes on ice (if not otherwise stated), centrifuged and applied via a $100 \mu \mathrm{l}$ sample loop onto the Superdex 75 column equilibrated with the same buffer. Elution was performed at a flow rate of $1 \mathrm{ml} /$ minute. The relative amounts of monomers and dimers were calculated by the integrated areas (mAU*min) of the corresponding protein peaks.

For determining the dimer dissociation constant $K_{\mathrm{d}}$ we used the equation:

$$
K_{\mathrm{d}}=[\text { monomer }]^{2} /[\text { dimer }]=\left(c_{\mathrm{t}}-x\right)^{2} / 0.5 x
$$

where $c_{\mathrm{t}}$ denotes the total concentration of subunits and $x$ is the concentration of dimer-forming subunits.

\section{Acknowledgements}

We thank our colleagues in the respective laboratories for their various contributions. Dr Holger Bauer made the DmTrx expression clone available to us. The work was supported by the DFG research focus "Selenoproteins" (grants Be 1540/6-3 and Wa 1126/2-2) as well as by the MaxPlanck-Society.

\section{References}

1. Arner, E. S. J. \& Holmgren, A. (2000). Physiological functions of thioredoxin and thioredoxin reductase. Eur. J. Biochem. 267, 6102-6109.

2. Powis, G., Kirkpatrick, D. L., Angulo, M. \& Baker, A. (1998). Thioredoxin redox control of cell growth and death and the effects of inhibitors. Chem. Biol. Interact. 111-112, 23-34.

3. Powis, G., Mustacich, D. \& Coon, A. (2000). The role of the redox protein thioredoxin in cell growth and cancer. Free Rad. Biol. Med. 29, 312-322.
4. Das, K. C. (2004). Thioredoxin system in premature and newborn biology. Antiox. Redox Signal. 6, 177-184.

5. Holmgren, A. (2000). Antioxidant function of thioredoxin and glutaredoxin systems. Antiox. Redox Signal. 2, 811-820.

6. Williams, C. H., Arscott, L. D., Müller, S., Lennon, B. W., Ludwig, M., Wang, P. F. et al. (2000). Thioredoxin reductase. Two modes of catalysis have evolved. Eur. J. Biochem. 267, 6110-6117.

7. Kanzok, S. M., Fechner, A., Bauer, H., Ulschmid, J. K., Müller, H.-M., Botella-Munoz, J. et al. (2001). Substitution of the thioredoxin system for glutathione reductase in Drosophila melanogaster. Science, 291, 643-646.

8. Bauer, H., Gromer, S., Urbani, A., Schnolzer, M., Schirmer, R. H. \& Müller, H. M. (2003). Thioredoxin reductase from the malaria mosquito Anopheles gambiae. Eur. J. Biochem. 270, 4272-4281.

9. Kanzok, S. M., Schirmer, R. H., Turbachova, I., Iozef, R. \& Becker, K. (2000). The thioredoxin system of the malaria parasite Plasmodium falciparum. Glutathione reduction revisited. J. Biol. Chem. 275, 40180-40186.

10. Friemann, R., Schmidt, H., Ramsawamy, S., Forstner, M., Krauth-Siegel, R. L. \& Eklund, H. (2003). Structure of thioredoxin from Trypanosoma brucei brucei. FEBS Letters, 554, 301-305.

11. Missirlis, F., Ulschmidt, J. K., Hirosawa-Takamori, M., Grönke, S., Schäfer, U., Becker, K. et al. (2002). Mitochondrial and cytoplasmic thioredoxin reductase variants encoded by a single Drosophila gene are both essential for viability. J. Biol. Chem. 277, 11521-11526.

12. Svensson, M. J., Chen, D. J., Pirotta, V. \& Larsson, J. (2003). The thioredoxinT and deadhead gene pair encode testis- and ovary-specific thioredoxins in Drosophila melanogaster. Chromosoma, 112, 133-143.

13. Bauer, H., Kanzok, S. M. \& Schirmer, R. H. (2002). Thioredoxin-2 but not thioredoxin-1 is a substrate of thioredoxin peroxidase-1 from Drosophila melanogaster: isolation and characterization of a second thioredoxin in D. melanogaster and evidence for distinct biological functions of Trx-1 and Trx-2. J. Biochem. 277, 17457-17463.

14. Ma, Q., Guo, C., Barnewitz, K., Sheldrick, G. M., Soling, H. D., Uson, I. \& Ferrari, D. M. (2003). Crystal structure and functional analysis of Drosophila Wind, a protein-disulfide isomerase-related protein. J. Biol. Chem. 278, 44600-44607.

15. Wollman, E. E., d'Auriol, L., Rimsky, L., Shaw, A., Jacquot, J. P., Wingfield, P. et al. (1988). Cloning and expression of a cDNA for human thioredoxin. J. Biol. Chem. 263, 15506-15512.

16. Pekkari, K., Gurunath, R., Arner, E. J. S. \& Holmgren, A. (2000). Truncated thioredoxin is a mitogenic cytokine for resting human peripheral blood mononuclear cells and is present in human plasma. J. Biol. Chem. 275, 37474-37480.

17. Miranda-Vizuete, A., Ljung, J., Damdimopoulos, A. E., Gustafsson, J. A., Oko, R., Markku, P. H. \& Spyrou, G. (2001). Characterization of Sptrx, a novel member of the thioredoxin family specifically expressed in human spermatozoa. J. Biol. Chem. 276, 31567-31574.

18. Jimenez, A. \& Miranda-Vizuete, A. (2003). Purification and characterization of delta3Trx-1, a splicing variant of human thioredoxin-1 lacking exon 3. Protein Expr. Purif. 27, 319-324.

19. Damdimopoulos, A. E., Miranda-Vizuete, A., PeltoHuikko, M., Gustafsson, J. A. \& Spyrou, G. (2002). 
Human mitochondrial thioredoxin. Involvement in mitochondrial membrane potential and cell death. J. Biol. Chem. 277, 33249-33257.

20. Miranda-Vizuete, A., Sadek, C. M., Jimenez, A., Krause, W. J., Sutovsky, P. \& Oko, R. (2004). The mammalian testis-specific thioredoxin system. Antiox. Redox Signal. 6, 25-40.

21. Holmgren, A. (1995). Thioredoxin structure and mechanism: conformational changes on oxidation of the active-site sulfhydryls to a disulfide. Structure, 3, 239-243.

22. Weichsel, A., Gasdaska, J. R., Powis, G. \& Montfort, W. R. (1996). Crystal structures of reduced, oxidized, and mutated human thioredoxins: evidence for a regulatory homodimer. Structure, 4, 735-751.

23. Haendeler, J., Hoffmann, J., Tischler, V., Berk, C. B., Zeiher, A. M. \& Dimmeler, S. (2002). Regulation of telomerase activity and anti-apoptotic function by protein-protein interaction and phosphorylation. Nature Cell Biol. 4, 473-479.

24. Casagrande, S., Bonetto, V., Fratelli, M., Gianzza, E., Eberini, I., Massignan, T. et al. (2002). Glutathionylation of human thioredoxin: a possible crosstalk between the glutathione and thioredoxin systems. Proc. Natl Acad. Sci. USA, 99, 9745-9749.

25. Watson, W. H., Pohl, J., Montfort, W. R., Stuchlik, O., Reed, M. S., Powis, G. \& Jones, D. P. (2003). Redox potential of human thioredoxin 1 and identification of a second dithiol/disulfide motif. J. Biol. Chem. 278, 33408-33415.

26. Ren, X., Björnstedt, M., Shen, B., Ericson, M. L. \& Holmgren, A. (1993). Mutagenesis of structural halfcystine residues in human thioredoxin and effects on the regulation of activity by selenodiglutathione. Biochemistry, 32, 9701-9708.

27. Andersen, J. F., Sanders, D. A., Gasdaska, J. R., Weichsel, A., Powis, G. \& Montfort, W. R. (1997). Human thioredoxin homodimers: regulation by $\mathrm{pH}$, role of aspartate 60, and crystal structure of the aspartate $60 \rightarrow$ asparagine mutant. Biochemistry, 36, 13979-13988.

28. Eklund, H., Gleason, F. K. \& Holmgren, A. (1991). Structural and functional relations among thioredoxins of different species. Proteins: Struct. Funct. Genet. 11, 13-28.

29. Lennon, B. W., Williams, C. H., Jr \& Ludwig, M. L. (2000). Twists in catalysis: alternating conformations of Escherichia coli thioredoxin reductase. Science, 289, 1190-1194.

30. Sandalova, T., Zhong, L., Lindqvist, Y., Holmgren, A. \& Schneider, G. (2001). Three-dimensional structure of a mammalian thioredoxin reductase: implications for mechanism and evolution of a selenocysteinedependent enzyme. Proc. Natl Acad. Sci. USA, 98, 9533-9538.
31. Katti, S. K., LeMaster, D. M. \& Eklund, H. (1990). Crystal structure of thioredoxin from Escherichia coli at 1.68 A resolution. J. Mol. Biol. 212, 167-184.

32. Capitani, G., Markovic-Housley, Z., DelVal, G., Morris, M., Jansonius, J. N. \& Schurmann, P. (2000). Crystal structures of two functionally different thioredoxins in spinach chloroplasts. J. Mol. Biol. 302, 135-154.

33. Dyson, H. J., Gippert, G. P., Case, D. A., Holmgren, A. \& Wright, P. E. (1990). Three-dimensional solution structure of the reduced form of Escherichia coli thioredoxin determined by nuclear magnetic resonance spectroscopy. Biochemistry, 29, 4129-4136.

34. Qin, J., Clore, G. M. \& Gronenborn, A. M. (1994). The high-resolution three-dimensional solution structures of the oxidized and reduced states of human thioredoxin. Structure, 2, 503-522.

35. Qin, J., Clore, G. M., Kennedy, W. M., Huth, J. R. \& Gronenborn, A. M. (1995). Solution structure of human thioredoxin in a mixed disulfide intermediate complex with its target peptide from the transcription factor NF kappa B. Structure, 3, 289-297.

36. Qin, J., Clore, G. M., Kennedy, W. M., Kuszewski, J. \& Gronenborn, A. M. (1996). The solution structure of human thioredoxin complexed with its target from Ref-1 reveals peptide chain reversal. Structure, 4, 613-620.

37. Menchise, V., Corbier, C., Didierjean, C., Saviano, M., Benedetti, E., Jacquot, J. P. \& Aubry, A. (2001). Crystal structure of the wild-type and D30A mutant thioredoxin $\mathrm{h}$ of Chlamydomonas reinhardtii and implications for the catalytic mechanism. Biochem. J. 359, 65-75.

38. Gronenborn, A. M., Clore, G. M., Louis, J. M. \& Wingfield, P. T. (1999). Is human thioredoxin monomeric or dimeric? Protein Sci. 8, 426-429.

39. Liepinsh, E., Baryshev, M., Sharipo, A., IngelmanSundberg, M., Otting, G. \& Mkrtchian, S. (2001). Thioredoxin fold as homodimerization module in the putative chaperone ERp29: NMR structures of the domains and experimental model of the $51 \mathrm{kDa}$ dimer. Structure, 9, 457-471.

40. Irmler, A., Bechthold, A., Davioud-Charvet, E., Hofmann, V., Réau, R., Gromer, S. et al. (2002). Disulfide reductases-current developments. Flavins Flavoproteins, 14, 803-815.

41. Otwinowski, Z. \& Minor, W. (1996). Processing of X-ray diffraction data collection in oscillation mode. Methods Enzymol. 276, 307-326.

42. Collaborative Computational Project, Number 4. (1994). The CCP4 suite: programs for protein crystallography. Acta Crystallog. sect. D, 50, 760-763.

43. Brunger, A. T., Adams, P. D., Clore, G. M., DeLano, W. L., Gros, P., Grosse-Kunstleve, R. W. et al. (1998). Crystallography \& NMR system: a new software suite for macromolecular structure determination. Acta Crystallog. sect. D, 54, 905-921.

Edited by P. Wright

(Received 17 September 2004; received in revised form 1 November 2004; accepted 2 November 2004) 